\title{
Artificial Intelligence Education System Based on Differential Evolution Algorithm to Optimize SVM
}

\author{
Weilin Long ${ }^{1}$ and Yi Gao $\mathbb{D}^{2}$ \\ ${ }^{1}$ Tianjin Modern Vocational Technology College, Tianjin 300350, China \\ ${ }^{2}$ College of Information Technical Science, Nankai University, Tianjin 300071, China \\ Correspondence should be addressed to Yi Gao; gaoyi@nankai.edu.cn
}

Received 15 November 2021; Accepted 13 December 2021; Published 7 January 2022

Academic Editor: Baiyuan Ding

Copyright (c) 2022 Weilin Long and Yi Gao. This is an open access article distributed under the Creative Commons Attribution License, which permits unrestricted use, distribution, and reproduction in any medium, provided the original work is properly cited.

\begin{abstract}
The artificial intelligence education system promotes the rooting of artificial intelligence in the education field and accelerates its entry into the era of intelligent education. This article focuses on the development of the artificial intelligence education system and proposes an artificial intelligence education system based on differential evolution algorithm optimization support vector machine. First, the processing of educational demand information data is automated, then a differential evolution algorithm is built to optimize the support vector machine model, and the model is used to implement various educational tasks to achieve automated education. The test results show that the model classification accuracy, classification recall rate, classification accuracy rate, and F1-score value are 4 items. Performances have been improved to improve the efficiency of education work and provide a reference for exploring the application and practice of artificial intelligence in education.
\end{abstract}

\section{Introduction}

As the core driving force of technological progress, artificial intelligence has brought profound changes to social development. The education field is also actively exploring how to deeply integrate with artificial intelligence, promote educational innovation, and build a new education ecology in the intelligent era. However, the specific empowerment of artificial intelligence in education is not achieved overnight [1-4]. The education field is currently facing problems such as lack of mature application models, lack of artificial intelligence experts, and lack of technical platform support. Artificial intelligence has not been able to effectively support the construction of educational tools and education systems [5-10]. How to promote the application of artificial intelligence and realize the convenient development of artificial intelligence systems will be an important link in the development of educational artificial intelligence [11-16].

Automation is a sign of the progress of human civilization and social modernization. With the progress of human society, automation technology continues to develop under the impetus of social demand. At present, the construction of artificial intelligence systems through automated methods is an important direction that the field of artificial intelligence has begun to pay attention to $[17,18]$. As a new research method, artificial intelligence methods such as automated machine learning and automated deep learning use the design idea of "training artificial intelligence with artificial intelligence" to expand the scope of artificial intelligence research and application and achieve the goal of open and inclusive artificial intelligence. The user only needs to provide the input data and task type, and the professional tasks such as algorithm and model construction in the system modeling are automatically completed by the machine, thereby effectively reducing the threshold of artificial intelligence applications and system development and promoting the standardization and module of artificial intelligence applications and automation [19-25].

The current research on artificial intelligence in education generally presents the status quo that there are many theoretical discussions and few practical applications [26, 27]. Automated artificial intelligence methods provide ideas for 
artificial intelligence research and applications in the education field. Based on the analysis of existing research and problems in the design and application of educational artificial intelligence systems, this paper discusses design methods based on automation ideas and proposes an artificial intelligence education system based on differential evolution (DE) algorithm optimization support vector machine (SVM). The educational demand information is processed automatically, and then a feature model is generated, and the model is used to implement various educational tasks so as to realize automatic education, improve the efficiency of educational work, and provide a reference for exploring the application and practice of educational artificial intelligence.

\section{Function Analysis of Artificial Intelligence Education System}

2.1. Function Frame Structure. The development function of the educational artificial intelligence application system includes five modules: educational needs, educational data, educational features, intelligent models, and educational applications. It mainly organizes relevant data and information according to the needs of educational work, digs out important information from them, uses the extracted educational features information, builds an intelligent education model, and invests it in education. Figure 1 shows the system function frame structure.

Educational Needs. Educators and related technical personnel are responsible for related work, setting up related issues according to the current requirements for educational work, and listing educational needs information.

Educational Data. Educators and related technical personnel are responsible for related work, collecting educational data related to this information, and using data processing technology to complete data cleaning and conversion operations based on educational demand information.

Educational Characteristics. Educational experts and related technical personnel are responsible for related work, extracting educational characteristics from the collated data information, and constructing an educational characteristics system at the same time.

Intelligent Model. Relevant technical personnel are responsible for related work, constructing intelligent models, and training them, according to the model evaluation results, optimizing the current model design plan to obtain the best model.

Educational Application. Educators and related technical personnel are responsible for related work and putting the optimized model into use, so as to play the role of the educational intelligence system.

2.2. Problems in Current System Development. At present, China has invested a lot of research funds for the development of educational artificial intelligence systems and encouraged major scientific research institutions to participate in the research of such projects. Although some research results have been obtained, there are still many problems:

(1) Technical Aspects. The system has poor portability, relying on manual debugging and selecting the corresponding operation mode according to the operation requirements. These control technologies are difficult to meet the needs of the model.

(2) System Development Cost Issue. System development requires a lot of money. In addition to the purchase and leasing of system hardware and software-related equipment, it also includes operation and maintenance, human resources, and other costs. Judging from the current development situation, the system development and operating costs have exceeded the expected range.

(3) System Development Complexity. There are many people involved in system development, involving experts and staff in multiple fields. In order to ensure the effective connection of various links, almost every link requires the participation of all staff, which increases the complexity of the system development.

(4) System Operation Limitation and Efficiency Issues. Most of the systems currently developed only support the current parameter setting requirements. If the data or educational features are changed, the system operating procedures need to be readjusted, and some hardware devices should be replaced according to the needs, resulting in the system application range restricted. In the test, it was found that the operating efficiency of the system was low and the mature system development plan was lacking.

\section{Design of Artificial Intelligence Education System Based on DE-SVM}

3.1. DE. DE is a random search heuristic technology based on group differences. It is a new and efficient technology. The DE algorithm is developed on the basis of genetic algorithm. Similar to the genetic algorithm, it has operations such as mutation, crossover, and selection.

The detailed process of the DE algorithm is as follows.

3.1.1. DE Algorithm Initializes the Population. To determine the population size $m$, each individual is an $n$-dimensional vector, and the initial population can be expressed as the following formula:

$$
X_{t}(0)=X_{t 1}(0)+X_{t 2}(0)+\cdots+X_{t j}(0) .
$$

Among them, $X_{t}(0)$ is the $t$-th individual of the 0 th generation and $X_{t j}(0)$ is the $j$-th gene of the $t$-th individual of the 0 th generation, $t=1,2, \ldots, m$. 


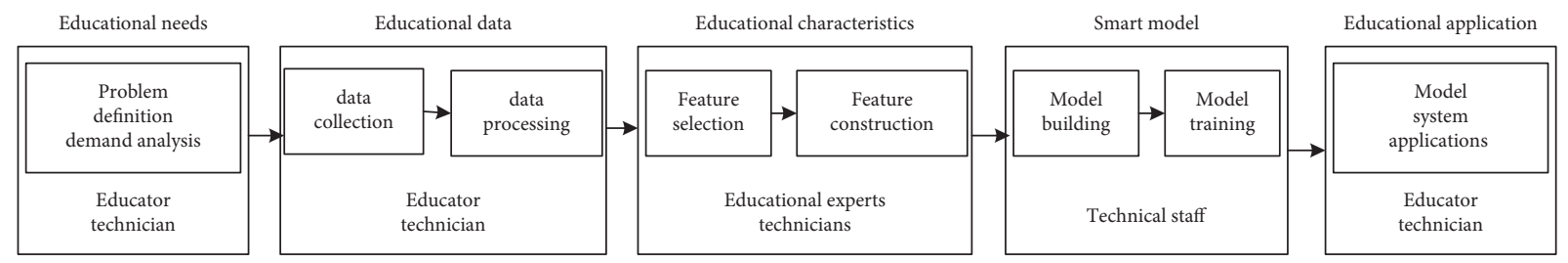

FIgURE 1: The functional framework structure of the artificial intelligence education system.

3.1.2. DE Algorithm Mutation. In the $k$ th iteration, for an individual $X_{t}(k)=\left(X_{\mathrm{t} 1}\left(k+X_{t 2}(k)+\ldots+X_{t n}(k)\right)\right.$, an intermediate vector $\operatorname{Lt}(k)$ is generated as follows and one is randomly selected from the population. For three individuals $X_{y 1}(k), X_{y 2}(k)$, and $X_{y 3}(k)$ and $y 1 \neq y 2 \neq y 3$, there are the following formulas:

$$
F_{t}(k)=X_{y 1}(k)-E *\left(X_{y 2}(k)-X_{y 3}(k)\right),
$$

where $\Delta=X_{y 2}(k)-X_{y 3}(k)$ is the difference vector.

$E$ is the scaling factor, used to control the influence of the difference vector, generally between $[0,1]$.

It can be seen intuitively to construct the variation vector $F t(k)$ in the two-dimensional parameter space as shown in Figure 2.

3.1.3. DE Algorithm Crossover Operation. In the $k$ th iteration, each individual crosses the first intermediate vector generated by it. Specifically, for each identical component, the first intermediate vector is selected with a certain probability and the second intermediate vector is generated. Generate the second intermediate vector $S_{t}(k)$, and calculate each component of $S_{t}(k)$ according to the following formula:

$$
S_{t j}(k)= \begin{cases}F_{t j}(k), & \text { rand }(0,1) \leq P, \\ X_{t j}(k), & \text { else, }\end{cases}
$$

where $P$ is the crossover probability, $F_{t j}(k)$ is a gene in the number one intermediate vector, and $X_{t j}(k)$ is a gene in the original individual.

3.1.4. DE Algorithm Selection Operation. The selection of the differential evolution algorithm adopts the greedy mode. In this process, according to the value of the fitness function, in the $k$ th iteration, the second intermediate vector $S_{t}(k)$ and the original vector $X_{t}$ of each individual are selected for the next generation $(k)$ the one with higher fitness, so that the population evolves to the optimal solution; the selection method is as follows:

$$
X_{t}(k+1)= \begin{cases}S_{t}(k), & f\left(S_{t}(k)>X_{t}(k)\right), \\ X_{t}(k), & \text { else. }\end{cases}
$$

After completing this step, continue to loop through the differential evolution operation until the number of iterations or fitness function requirements are met, and then the algorithm stops.

3.2. SVM. SVM is a supervised learning model that can analyze data between classification and regression analysis.
Its basic idea is to define a linear classifier with the largest interval in the function space. The SVM classifier also includes a kernel technique that allows nonlinear classification. The learning strategy of the SVM classifier is the optimal classification hyperplane, where this hyperplane must meet the classification requirements to maximize the blank space on both sides of the hyperplane while ensuring the classification accuracy. The main idea of SVM is as follows: given a set of data set $T=\left\{\left(x_{-} 1, y_{-} 1\right),\left(x_{-} 2, y_{-} 2\right), \ldots\right.$, $\left.\left(x \_N, y \_N\right)\right\}$, where $x \_i \in R n, y \_i \in\{-1,+1\}, i=1,2, \ldots, N$, which satisfy

$$
y_{i}\left(w \cdot x_{i}+b\right) \geq 1
$$

making

$$
\min _{w, b} \frac{w^{2}}{2}
$$

According to Lagrangian duality, the optimal solution can be obtained by solving the dual problem of the original problem, which is transformed into

$$
\begin{cases}\max _{\alpha} & \left(\frac{1}{2} \sum_{i=1}^{N} \sum_{j=1}^{N} \alpha_{i} \alpha_{j} y_{i} y_{j}\left(x_{i} \cdot x_{j}\right)+\sum_{j=1}^{N} \alpha_{i}\right) \\ \text { s.t. } & \sum_{j=1}^{N} \alpha_{i} y_{i}=0, \quad \alpha_{i} \geq 0, i=1,2, \ldots, N .\end{cases}
$$

After adding a minus sign to the target formula, the problem of solving the maximum value is converted into a minimum value problem, and after conversion, it becomes

$$
\begin{cases}\min _{\alpha} & \left(\frac{1}{2} \sum_{i=1}^{N} \sum_{j=1}^{N} \alpha_{i} \alpha_{j} y_{i} y_{j}\left(x_{i} \cdot x_{j}\right)+\sum_{j=1}^{N} \alpha_{i}\right) \\ \text { s.t. } & \sum_{j=1}^{N} \alpha_{i} y_{i}=0, \quad \alpha_{i} \geq 0, i=1,2, \ldots, N .\end{cases}
$$

After calculating the solution $\alpha$, we further solve $w$ and $b$ according to $\alpha$ to obtain the maximum separation hyperplane and the classification decision function.

3.3. SVM Optimization Process Based on DE Algorithm. The function of the DE algorithm is to optimize the parameters of the SVM, that is, to find the optimal parameter combination (C, gamma), so that the SVM model has the best classification performance. The detailed steps of SVM 


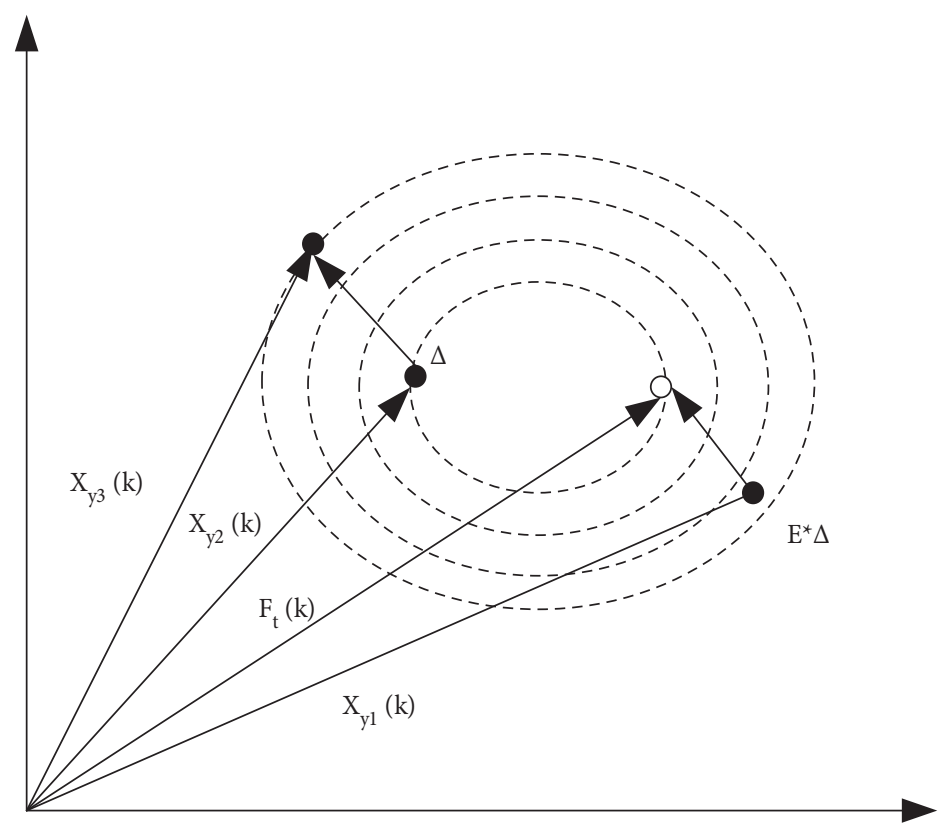

FIGURE 2: Schematic diagram of mutation vector construction.

parameter optimization based on the DE algorithm are as follows:

Step 1. Define the optimization objective function and initialize the size of the population $N$, the scaling factor $k$, the evolution algebra $g$, the crossover probability $\mathrm{CR}$, the end condition, and the range of the parameters $\mathrm{C}$, and gamma to randomly generate a combination $(\mathrm{C}$, gamma).

Step 2. Use the current parameter combination (C, gamma) as the SVM parameter, use the SVM model to train and test the sample data, and obtain the classification result.

Step 3. Compare the above classification results with the actual classification results, calculate the value of the objective function, and judge whether the value reaches the predetermined accuracy or the maximum evolutionary algebra. If any of them is satisfied, go to Step 8; otherwise, $g=g+1$; enter the next generation of evolution.

Step 4. Randomly select 4 different individuals $X_{i}(g)$ from the population of the current $g$ generation and perform the mutation operation on the individuals according to formula (2) to generate the variant individual $V_{i}(g+1)$ of the $g+1$ generation.

Step 5. Perform crossover operation on individual $V_{i}(g+1)$ according to formula (3) to generate the experimental individual $U_{i}(g+1)$ of the $g+1$ generation.

Step 6. Perform a greedy selection on the experimental individual $U_{i}(g+1)$ of the $g+1$ generation according to formula (4) and generate the individual $X_{i}(g+1)$ of the $g+1$ generation.

Step 7. Calculate and generate a new parameter combination (C, gamma) in the population of the $g+1$ generation and then go to Step 2.
Step 8. Obtain the optimal parameter combination (C, gamma) of the SVM and then use the DE-SVM model to train and test the sample data to apply it to the passenger flow transfer model. The detailed process of using the DE algorithm to optimize SVM parameters is shown in Figure 3.

3.4. Model Building of Artificial Intelligence Education System. This system is based on self-contained thinking and learning theory. It automatically processes education demand information in the cloud and then generates a feature model, which is used to implement various educational tasks, thereby realizing automated education and improving the efficiency of educational work. Among them, model training is the key to improve educational work. Based on data features, it automatically combines educational information to create interpretable features. When the characteristics of education change, the education model is automatically adjusted to improve the efficiency of the system and expand the scope of system application conditions. The system model design scheme is shown in Figure 4.

The system model is mainly composed of 5 parts, namely, demand analysis and problem definition, education data, automation platform, application interface, and education system. Among them, the demand analysis and problem definition module is the extraction of intelligent education information and the summary of the problem as the input port of system operation data. The education data module is to extract important information about the development of education from the first section, as an information extraction and transmission tool, and send this part of the information to the automation platform. It completes various operations in the cloud. These operations all adopt automated processing technologies to clean up 


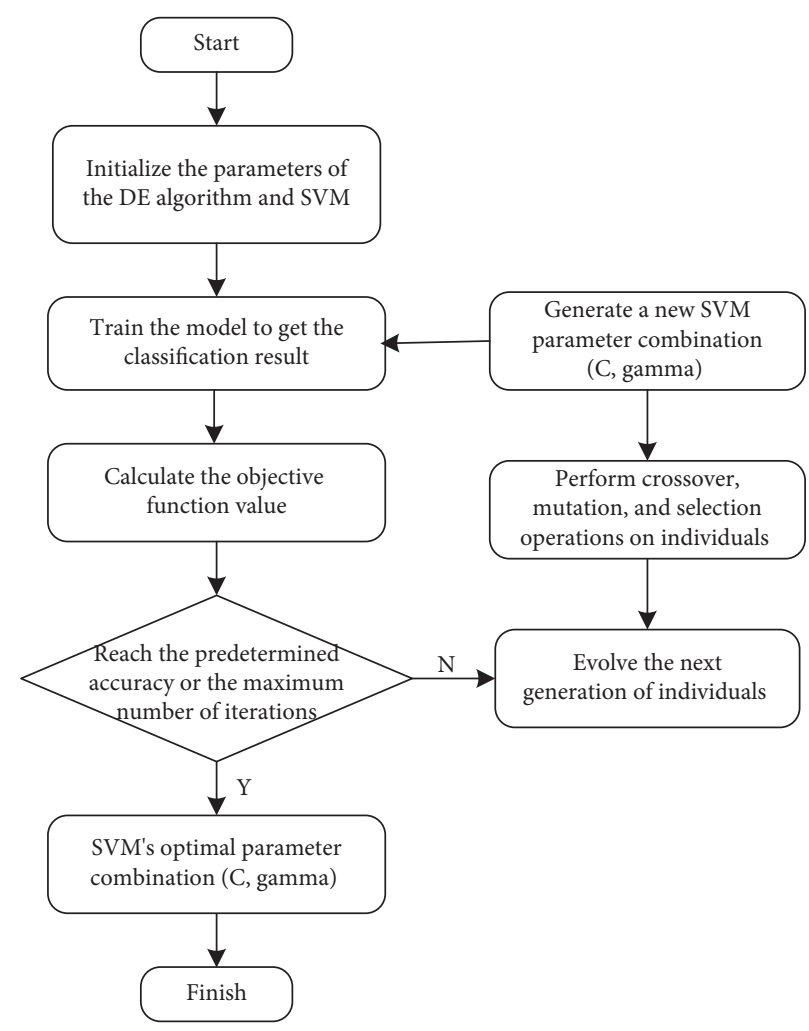

Figure 3: Flow chart of the DE-SVM model.

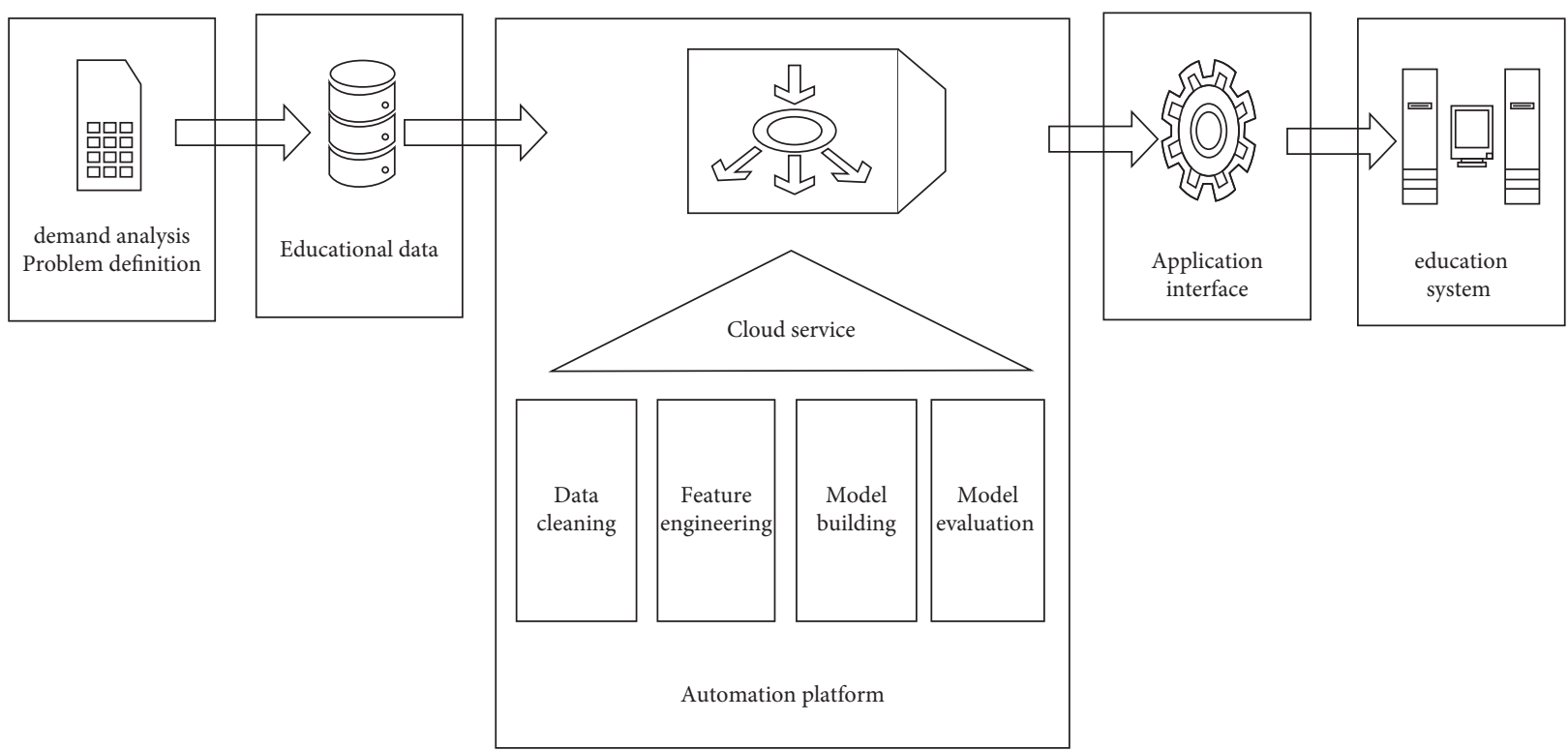

Figure 4: Artificial intelligence education system model.

data, create feature projects, build automated education models, and evaluate the role and performance of the models. The communication connection between the automation platform and the education system is established through the application interface. Through this interface, the automation platform operation status information is sent to the education system. According to the changes in the educational characteristics, the education system adjusts the operating parameters of the automation platform and completes the automatic update in the cloud.

\section{Case Application Analysis}

This research applies the system model to the MOOC and collects a large amount of data about the MOOC to provide a data source for system testing and analysis. Among them, 
TABLE 1: Forecast results.

\begin{tabular}{lcccc}
\hline Number & $\begin{array}{c}\text { Accuracy } \\
(\%)\end{array}$ & $\begin{array}{c}\text { Recall rate } \\
(\%)\end{array}$ & $\begin{array}{c}\text { Accuracy rate } \\
(\%)\end{array}$ & $\begin{array}{c}F 1 \text {-score } \\
(\%)\end{array}$ \\
\hline 1 & 91.42 & 92.32 & 93.52 & 91.55 \\
2 & 91.56 & 92.45 & 93.64 & 91.58 \\
3 & 91.67 & 92.61 & 93.68 & 91.67 \\
\hline
\end{tabular}

data information mainly includes learning process, learning experience, and learning evaluation. This system mainly provides convenient conditions for MOOC study and discussion. With the help of teaching assistants and teachers, different types of text data were marked to form 4 types of information, namely, personal feelings, learning evaluation, content goals, and curriculum questions.

$$
F-\text { score }=\left(1+\beta^{2}\right) \frac{\text { accuracy } \times \text { recall rate }}{\beta^{2} \times \text { accuracy }+ \text { recall rate }} .
$$

The test indicators for model job performance include four items: information classification accuracy, classification recall rate, classification accuracy rate, and classification $F$ value ( $F 1$-score). There are about 6,000 courses forum data, of which $80 \%$ are used as the training set, $10 \%$ of the system operation verification set, and the remaining $10 \%$ are used as evaluation data support. These data are brought into the system created in this article. The indicators of the currently developed system are around $70 \%$, and there is no more than $80 \%$. If the test result of this system is more than $80 \%$, it is considered that this system is better than the system developed in the past. Performance has improved. The predicted results of various indicators are shown in Table 1.

Judging from the above three sets of test results, the operating performance of this model is better than that of the traditional system. The values of all indicators are more than $91 \%$, and the classification accuracy rate is more than $93 \%$.

\section{Result}

This article focuses on the development of the education artificial intelligence system. Aiming at the problems of current system development, from the perspectives of operating efficiency, cost, application range, and so on, this paper proposes an artificial intelligence education system based on the differential evolution algorithm to optimize SVM. First, the processing of education demand information is automated, then a feature model is generated, and the model is used to implement various educational tasks to achieve automated education. The test results show that the model classification accuracy, classification recall rate, classification accuracy rate, and $F 1$-score value are 4 items. The performance has been improved to improve the efficiency of education work and provide a reference for exploring the application and practice of artificial intelligence in education. Automated methods provide directions to promote the application of artificial intelligence in education. Combining educational scenarios and educational needs, the practice of smart education applications should be the focus of the next step of research.

\section{Data Availability}

The dataset used to support the finding of this study can be accessed upon request.

\section{Conflicts of Interest}

The authors declare that there are no conflicts of interest.

\section{References}

[1] D. Liu, "Application scenario analysis and system design of intelligent assistants in education," China Audio-Visual Education, vol. 392, no. 9, pp. 27-35, 2019.

[2] Z. Zhang, L. Zhang, and Q. Luo, "Actual analysis of artificial intelligence education application: methods and limits of teaching automation," China Distance Education, vol. 530, no. 3, pp. 5-17, 2019.

[3] Q. Sun and X. Li, "Research on the design of self-education system of resource intelligence course," Research in AudioVisual Education, vol. 6, pp. 98-104, 2019.

[4] P. Wang, "Application analysis and design of artificial intelligence in educational videos," Audio-Visual Education Research, vol. 323, no. 3, pp. 95-102, 2020.

[5] J. Zhao, X. Xue, and C. Xu, "Review of the application of a new generation of artificial intelligence technology in power system scheduling operation," Automation of Electric Power Systems, vol. 694, no. 24, pp. 6-15, 2020.

[6] J. Wang and X. Qu, In-depth Understanding of AutoML and AutoDL: Building an Automated Machine Learning and Deep Learning Platform, Machinery Industry Press, Beijing, China, 2019.

[7] L. WuQ. Liu et al., "Research on the semantic analysis model and application of MOOC comments from the perspective of big data," Audio-visual Education Research, vol. 38, no. 11, pp. 43-48, 2017.

[8] M. Shen, G. Cen, W. Zhou, R. Zhu, and Y. Liang, "Early education system design based on CNN intelligent AI assistant," Journal of Zhejiang University of Science and Technology, vol. 32, no. 6, pp. 590-594, 2020.

[9] S. Jiang, Application of Deep Learning in Student Education Recommendation System, Changchun University of Technology, Changchun, China, 2020.

[10] X. Yu, "The application of automated methods in the design of educational artificial intelligence systems," Electronic Technology and Software Engineering, vol. 17, pp. 186-187, 2021.

[11] Y. Liu, "Research on the application of artificial intelligence industry based on big data in education innovation," in Proceedings of the 18th Shenyang Science Conference, p. 8, Shenyang, China, October 2021.

[12] G. Li, "A performance prediction decision-making system using machine learning technology under the background of smart education," Journal of Ningde Normal University (Natural Science Edition), vol. 33, no. 1, pp. 36-41, 2021.

[13] W. Ma and J. Ma, "The application and prospect of artificial intelligence in the field of physical education," Journal of Sports Adult Education, vol. 36, no. 6, pp. 42-45+99, 2020.

[14] Q. Hu, Design and Performance Optimization of AI Algorithm Education System for Educational Robots, Donghua University, Shanghai, China, 2020.

[15] W. Zhong and Z. Li, "Research on network education system based on machine learning," Journal of Communications, vol. 39, no. S1, pp. 135-140, 2018. 
[16] M. Yu, F. Xiang, and Z. Zhu, "Educational application and innovative exploration of machine learning in the perspective of artificial intelligence," Big Data Era, vol. 1, pp. 64-73, 2018.

[17] Z. Wang, "Analysis of the application of artificial intelligence in the field of education," China New Telecommunications, vol. 20, no. 22, p. 179, 2018.

[18] X. Yue, X. Cheng, and J. Li, "Research on "artificial intelligence + education" system based on big data," Computer Products and Circulation, vol. 5, p. 133, 2018.

[19] L. Shi, "Educational application of open source artificial intelligence system tensorflow," Modern Educational Technology, vol. 28, no. 1, pp. 93-99, 2018.

[20] E. Chen, L. Qi, S. Wang et al., "Key technologies and applications of adaptive learning for intelligent education," Journal of Intelligent Systems, vol. 1-13, 2021.

[21] W. Lan and Z. Li, "Thoughts on the teaching reform of the "database" course integrating the cultivation of computational thinking," Computer Age, vol. 11, pp. 110-116, 2020.

[22] S. Zhang, "Talking about the application of artificial intelligence based on expert system in the field of education," Digital World, vol. 3, p. 167, 2020.

[23] S. Zhang, "Exploration of the application of "AI+education" in the United States," Shanghai Information Technology, vol. 1, pp. 54-57, 2020.

[24] J. Ran, "The future has come-looking at artificial intelligence and education," Communication Power Technology, vol. 36, no. 11, pp. 169-171, 2019.

[25] W. Li, "Research on artificial intelligence and future education development under the background of new economy," Marketing Industry, vol. 47, pp. 79-80, 2019.

[26] A. Li, Z. Guo, S. Xie, D. Zhao, and S. Zhang, "Research on the classification of steel plate surface defects based on SVM with ant colony and particle swarm optimization," China Test, vol. 46, no. 1, pp. 110-116, 2020.

[27] C. Yun, S. Song, Y. Pan, and Y. Li, "Credit risk assessment model based on SVM hybrid integration," Computer Engineering and Applications, vol. 52, no. 4, pp. 115-120, 2016. 